Nouvelles perspectives en sciences sociales

Revue internationale de systémique complexe et d'études relationnelles

\title{
Littérature et communauté : vitalité et reconnaissance du Far Ouest francophone
}

\author{
Pamela V. Sing
}

Volume 8, numéro 2, mai 2013

Sur le thème de représentations identitaires et expressions culturelles de la francophonie canadienne à travers ses pratiques artistiques et médiatiques

URI : https://id.erudit.org/iderudit/1016473ar

DOI : https://doi.org/10.7202/1016473ar

Aller au sommaire du numéro

\section{Éditeur(s)}

Prise de parole

\section{ISSN}

1712-8307 (imprimé)

1918-7475 (numérique)

Découvrir la revue

Citer cet article

Sing, P. V. (2013). Littérature et communauté : vitalité et reconnaissance du Far Ouest francophone. Nouvelles perspectives en sciences sociales, 8(2), 119-144. https://doi.org/10.7202/1016473ar

\section{Résumé de l'article}

Pendant presque un siècle, la « littérature franco-albertaine » a signifié les ouvrages de principalement un écrivain reconnu. Jusqu'aux années 1950, ce fut l'écrivain d'origine française, George Bugnet, qui s'est établi en Alberta peu de temps après la création de la province. De 1960 jusqu'à la fin du siècle, ce fut Marguerite-A. Primeau, qui, née à Saint-Paul-des-Métis dans le nord de l'Alberta, s'est établie à Vancouver en 1954. Or, depuis l'an 2000, la francophonie albertaine connaît une croissance démographique, institutionnelle et culturelle qui permet de parler de sa vitalité. Au seul plan littéraire, le nombre de ses écrivains, définis dans cet article comme les francophones qui sont nés en Alberta ou bien qui, originaires d'une autre francophonie, vivent et écrivent ou ont vécu et ont écrit en Alberta, a augmenté. Ils sont encore peu nombreux relativement aux milieux franco-manitobaine, franco-ontarienne ou acadienne, mais dans l'unique cadre albertain, le « Far Ouest » du titre, « cela bouge ». On pourrait dire que la communauté franco-albertaine se trouve actuellement non tant préoccupée par sa survivance, que soucieuse de son épanouissement. Du coup, elle cherche la reconnaissance. Auprès de qui? Par quels moyens? Cet article tente d'apporter quelques éléments de réponse à ces questions tout en réclamant pour la production littéraire un rôle particulièrement important. 


\title{
Littérature et communauté : vitalité et reconnaissance du Far Ouest francophone
}

\author{
Pamela V. Sing \\ Campus St-Jean, \\ Université de l'Alberta, Edmonton
}

\section{Introduction}

Gi, pendant le XX $X^{\mathrm{e}}$ siècle, les francophones de l'Alberta, le « Far $\checkmark$ Ouest francophone » de mon titre, ont été l'objet de commentaires ou jugements disgracieux voulant qu'ils soient autant de "vaches mortes " ", de " dead ducks" " ou de " cadavres encore chauds ${ }^{3}$ ", ou bien encore, "les derniers Mohicans ${ }^{4}$ ", le tournant du $\mathrm{XXI}^{\mathrm{e}}$ siècle a apporté des développements qui pourraient faire taire les auteurs de telles prédictions. Au seul plan sociodémographique, les données publiées par Statistique

1 Voir Jacques Ferron, "La Vache morte au canyon ", dans Contes, édition intégrale, Montréal, Hurtubise HMH, 1985 [1953], p. 79-105.

2 René Lévesque, pendant une entrevue radiophonique diffusée à Ottawa en 1968 et dans un discours prononcé à Windsor, en Ontario, en 1969.

3 Le romancier québécois, Yves Beauchemin, a prononcé ces paroles en 1982, pendant la Commission Bélanger-Campeau.

4 Charles Castonguay, mathématicien et démographe à l'Université d'Ottawa, qui s'était prononcé dans les années 1970 au sujet de la disparition imminente des minorités francophones hors Québec, a repris ses propos d'ordre nécrologique vingt-neuf ans plus tard : "It must be great to be the last of the Mohicans. Imagine the subsidies! " dans "Getting the Facts Straight on French Reflections Following the 1996 Census ", Inroads, n 8, 1999, p. 69. 
Canada ${ }^{5}$ basées sur les résultats du Recensement de la population canadienne de 2011 à l'égard de la "présence du français " révèlent que c'est l'Alberta la province canadienne qui connaît le taux de croissance le plus élevé du pays : entre 2006 et 2011, le nombre d'individus ayant déclaré avoir le français comme langue maternelle a connu une hausse de $18 \%$, tandis que le nombre de ceux ayant déclaré parler le français le plus souvent à la maison a connu une hausse de $38 \%$. Il est vrai que la croissance en nombre absolu s'accompagne d'une baisse proportionnelle, mais cela n'a pas empêché l'Association canadienne-française de l'Alberta (ACFA) d'affirmer avec fierté dans un communiqué diffusé le 13 février 2013 que 238000 personnes vivant en Alberta disent parler le français et que le nombre continue de croître. Par ailleurs, l'émergence d'un domaine d'étude productif et dynamique, ayant pour objet la "Franco-Amérique ", c'est-àdire l'état actuel de l'entité connue jadis comme " l'Amérique française ", a contribué à faire ressortir la vitalité réelle de la francophonie albertaine - à tout le moins auprès des chercheurs et intellectuels spécialistes de la question des francophonies minoritaires au Canada. Car désormais, la pérennité des petites cultures francophones partout sur le continent nord-américain est perçue comme un phénomène digne d'intérêt ${ }^{6}$. Pour Dean Louder et Éric Waddell, les géographes qui ont forgé le terme "Franco-Amérique ", l'Ouest canadien est l'un des "foyers oubliés ${ }^{7}$ " de l'Amérique du Nord francophone, mais mis en rapport avec d'autres "foyers oubliés ", tel le Midwest américain où la participation à la Franco-Amérique prend la forme de traces ou de vestiges mnémoniques d'un passé francophone presque disparu, l'Ouest franco-canadien, y compris le Far Ouest, acquiert une incontestable épaisseur de réalité. Dans "À quoi sert la

5 Accessibles à : [en ligne] http://www12.statcan.gc.ca/census-recensement/2011/ as-sa/98-314-x/98-314-x2011003_1-fra.cfm, consulté le 26 février 2013.

6 Ce développement est dû principalement aux recherches et publications des géographes Dean Louder et Éric Waddell, auteurs, entre autres, du recueil d'études intitulé précisément Franco-Amérique, Sillery, Septentrion, 2008.

7 Voir la carte stylisée de la Franco-Amérique conceptualisée par Éric Waddell dans Dean Louder et Éric Waddell Franco-Amérique, p. 11. 
Franco-Amérique? ", le sociologue Joseph Yvon Thériault identifie l'Ouest canadien comme l'une "des collectivités francophones dont l'identité linguistique est forte ", mais c'est pour préciser aussitôt, dans la même phrase, qu'elle fait "difficilement communauté », car elle " a eu tendance dans les dernières années à délaisser sa dimension communautaire - son inscription dans la vieille intention nationale canadienne-française - pour se percevoir comme un groupement urbain, largement ouvert à la diversité cosmopolitique ${ }^{8} »$.

Cette dernière " observation " sert avant tout à faire ressortir le point auquel la francophonie en Alberta peut être méconnue par ceux qui s'intéressent à elle de loin. Car, de toute évidence, l'auteur ignore les efforts déployés, entre autres, par l'ACFA pour toujours renforcer les liens entre ses treize branches ou agences régionales d'une part et d'autre part, pour bâtir une communauté inclusive ${ }^{9}$ - en cela, elle est soutenue fortement par les activités et publications du Groupe de recherche sur l'inter/transculturalité et immigration (GRITI) du Campus Saint-Jean, le campus francophone de l'Université de l'Alberta - et par le Secrétariat francophone de l'Alberta pour appuyer activement les priorités en matière des services en français identifiées par la communauté (à travers les chefs de file de l'ACFA et des consultations communautaires), dont notamment celle de maintenir et d'accroître la visibilité de la communauté francophone et de la langue et de la culture françaises en Alberta. Voilà qui, à mon avis, affirme l'existence d'une francophonie soucieuse de conserver son caractère communautaire tout en assurant un dynamisme

8 Joseph Yvon Thériault, "À quoi sert la Franco-Amérique? ", dans Dean Louder et Éric Waddell (dir.), Franco-Amérique, p. 363. Je souligne.

9 L'ACFA a annoncé dans un communiqué diffusé le 26 février 2013 que son Conseil d'administration s'engage à "rassembl[er] toutes les instances de l'ACFA [... dans le but d'élaborer] un plan d'action qui répondra aux questions clés longuement débattues et qui ralliera les membres autour d'une vision commune d'un membership à valeur ajoutée, tout en répondant aux impératifs économiques des ACFA régionales, provinciale et du journal Le Franco ». Accessible à : [en ligne] http://www.acfa.ab.ca/Documents/ Communiques2010/20130226_Membership-de-1-ACFA-Vers-une-visioncommune.pdf, consulté le 26 février 2013. 
évolutif en tant que collectivité désireuse de vivre et de faire vivre sa pluralité.

\section{Quête de reconnaissance}

Ce qui m’intéressera particulièrement ici est l'objectif concernant la visibilité de la communauté et de sa francité. Certes, la communauté franco-albertaine elle-même, composée de ceux et celles qui s'identifient comme des francophones vivant en Alberta, qu'ils soient originaires de la province ou bien d'une autre francophonie, s'est toujours sentie consciente de sa francité, non seulement à l'époque où elle sentait une appartenance au Canada français, mais aussi après la Révolution tranquille, lorsque, par exemple, l'ACFA, en tant que porte-parole de la communauté, a mené des sondages auprès des siens pour connaître leur volonté concernant un changement d'étiquette identitaire ${ }^{10}$. Il s'avère, toutefois, qu'il ne lui suffit pas de se connaître. Il lui importe, de surcroît, d'être reconnue en dehors de ses frontières, et ce, non pas comme une composante indifférenciée de "l'Ouest», de " la prairie " ou " des prairies ", mais en tant qu'un groupe francophone ayant ses particularités, ses spécificités. Elle a des affinités avec les communautés francophones de la Saskatchewan et du Manitoba, mais elle ne s'en distingue pas moins et voudrait par conséquent que le caractère singulier de son identité culturelle soit reconnu. Et, de surcroît, valorisé. Tel que le souligne le philosophe Charles Taylor dans The Politics of Recognition, la diffusion répandue de l'idée que nous somme formés par la reconnaissance, que celle-ci forge l'identité, nous a conduits à considérer la méconnaissance comme un tort tout aussi grave que l'inégalité, l'exploitation ou l'injustice ${ }^{11}$.

$\overline{10}$ En 2006, l'ACFA a présenté à ses membres la possibilité de changer de nom. Certains, pensant aux nouveaux arrivants, cherchaient un nom plus inclusif tandis que d'autres cherchaient à éliminer la référence au Canada français, mais encore d'autres tenaient à expliciter leur canadianité. En fin de compte, l'ACFA a décidé de garder tel quel son nom.

11 Charles Taylor, The Politics of Recognition, Princeton, Presses Universitaires de Princeton, 1994, p. 64-65. 


\section{Littérature et reconnaissance}

Il en ressort la question de savoir comment une communauté en quête de reconnaissance peut s'y prendre. Dans la perspective adoptée ici, la littérature a un rôle central à y jouer. En 2005, la Fédération culturelle canadienne-française (FCCF) a affirmé que, "dans un contexte minoritaire, les activités artistiques et culturelles insufflent de la vie à une communauté, l'animent et définissent ses caractéristiques uniques que ce soit dans un contexte urbain ou rural ${ }^{12}$ ». Pour l'écrivaine franco-ontarienne Michèle Matteau, les artistes littéraires

sont la mémoire d'une communauté. Ils lui rappellent son histoire et les sentiers parcourus. Ils sont des vigies qui la scrutent et prennent conscience de sa mouvance et de ses dérives. Ils sont les sourciers qui dévoilent ses richesses et expriment ses possibles. Ils sont ces témoins qui la racontent aux autres et servent de liens entre les communautés. Ils sont des agents actifs de la vitalité de la langue française, des résistants résolus face à l'action uniformisante de la culture américaine ${ }^{13}$.

En fait, le lien entre la littérature conçue au sein d'une communauté et la communauté est encore plus fondamental que ce que n'en disent la FCCF et Michèle Matteau. Littérature et communauté sont interreliées dans un rapport d'implication réciproque. D'une part, l'œuvre littéraire travaille, transforme et (re)produit, (re)construit ou (ré)invente la communauté et participe ainsi de la façon dont celle-ci se perçoit, s'identifie. D'autre part, la communauté constitue le moment de reconnaissance de l'œuvre, en détermine la valeur et, ce faisant, favorise sa circulation dans d'autres communautés. La réception ou la reconnaissance d'une ouvre littéraire donnée entraine celle aussi de la communauté dont elle est issue.

12 Le passage, extrait d'un mémoire présenté au Comité permanent des finances de la Chambre des communes le 31 octobre 2005, est cité en exergue dans l'étude entreprise par le Commissariat aux langues officielles sur les mesures prises par le gouvernement fédéral en vue de promouvoir les arts et la culture des communautés de langue officielle en situation minoritaire; [en ligne] http://www.ocol-clo.gc.ca/html/stu_etu_032008_p2_f.php, consulté le 7 février 2013.

13 Michèle Matteau, "Où donc est la littérature? ", Liaison, n 134, 2006-07, p. 25. 


\section{Un concept théorique de la reconnaissance}

Un concept théorique de la reconnaissance productif pour la discussion des liens entre la littérature et la communauté francophone en situation minoritaire se trouve chez Paul Ricœur. Dans une étude traitant du développement de nouvelles histoires littéraires francophones d'Amérique, "issues de la reconnaissance par les communautés de l'existence d'une littérature qui leur est propre, différenciée de la littérature québécoise ${ }^{14}$ ", littéraire Micheline Cambron définit la littérature comme « la perlaboration de l'identité narrative d'une communauté15 ", précisant que cette identité narrative s'inscrit toujours dans ce que Ricœur nomme un "parcours de reconnaissance ${ }^{16}$ ". Ce parcours, résume-t-elle, est

composé d'un premier moment qui est celui de l'identification, d'un second moment qui consiste à inscrire cette reconnaissance dans un agir propre et donc à y puiser la définition d'un soi-même, et enfin d'un troisième moment qui place la reconnaissance de soi dans une relation de réciprocité avec l'autre ${ }^{17}$.

Chez Ricœur, les deuxième et troisième moments du parcours s'articulent en rapport avec un traitement du verbe " reconnaître " qui passe de la voix active à la voix passive. Au premier pôle, cela donne "Je reconnais telle chose, telle personne, tel objet ", ce qui est un acte exprimant une prétention d'exercer une maîtrise intellectuelle sur le champ des significations. Au pôle opposé, cela donne "Je désire être reconnu ", demande qui exprime une attente qui est possible à satisfaire uniquement dans une situation de reconnaissance mutuelle.

\section{De la reconnaissance auprès de qui?}

Adoptant toujours la perspective selon laquelle une telle réciprocité peut s'établir via la littérature, il faut poser la question

14 Micheline Cambron, "L'Enchevêtrement des histoires littéraires dans la francophonie d'Amérique vu à travers le renouvellement épistémologique de l'histoire littéraire ", Francophonies d'Amérique, n 26, 2008, p. 345.

15 Ibid., p. 346.

16 Paul Ricœur, Parcours de la reconnaissance : trois études, Paris, Gallimard, 2004.

17 Micheline Cambron, op. cit., p. 347. 
suivante : avec qui les écrivains francophones de l'Alberta pourraient-ils vraisemblablement chercher à former des liens de reconnaissance mutuelle? On serait sans doute nombreux à répondre que les meilleures possibilités résident parmi les lecteurs de tout le Canada francophone. Depuis la fragmentation du Canada français, les francophones de toutes les régions du Canada ont pris l'habitude de s'identifier de façon territoriale, mais ils n'ont pas pour autant perdu le désir de " faire communauté " avec toutes les communautés de l'ancien Canada français. Ils se doutent bien, toutefois, que si le Québec donne certains signes de sa volonté de reconnaitre ses liens historiques avec les autres francophonies du continent ${ }^{18}$, ces dernières n'ont pas toutes la même importance. Les écrivains francophones du Far Ouest en l'occurrence auront à accroître considérablement leur visibilité et leur pouvoir d'attraction, pour ainsi dire, avant que ces signes ne conduisent à un réel intérêt pour eux. En témoigne l'article au sujet des nouvelles histoires littéraires cité plus haut, dont l'auteure québécoise constate qu'il existe « des histoires (et des anthologies) des littératures acadienne, ontaroise, manitobaine, fransaskoise et, sans doute sous peu, britanno-colombienne ${ }^{19}$ ». L'exclusion dans cette énumération de la littérature franco-albertaine est probablement liée à l'inexistence jusqu'ici d'une anthologie portant exclusivement sur ladite littérature, mais aussi à l'ignorance des anthologies qui l'incluent, dont notamment Moissons $^{20}$, Sous les mâts de la prairie : anthologie littéraire fransaskoise et de l'Ouest canadien ${ }^{21}$ et Paroles francophones de l'Ouest et du Nord canadiens ${ }^{22}$. Pour être juste, je dois préciser

18 Voir à ce sujet, Pamela V. Sing, "Writing the Hinterland (Back) into the Heartland: the Franco-Canadian Farouest in Two Novels by Nicolas Dickner and D.Y. Béchard ", British Journal of Canadian Studies, vol. 24, n 2, 2011,

19 p. 221-246.

Ibid.

20 Roger Motut, Moissons, Edmonton, Ministère de l'Éducation de l'Alberta, $1979,226 \mathrm{p}$.

21 Bernard Wilhelm (dir.), Sous les mâts de la prairie : anthologie littéraire fransaskoise et de l'Ouest canadien, Regina, Éditions de la Nouvelle plume, 2000, 294 p.

22 Carol J. Harvey (dir.), Paroles francophones de l'Ouest et du Nord canadiens, Winnipeg, Presses universitaires de Saint-Boniface, 2012, xv, 221 p. 
que le dernier titre a paru en 2012, trop tard pour que l'auteure de l'article puisse en tenir compte... Quoi qu'il en soit, force est de conclure que dans la conjoncture socioculturelle actuelle, c'est auprès des francophones canadiens hors-Québec que les écrivains franco-albertains chercheraient le plus profitablement à forger des liens de reconnaissance mutuelle.

\section{Une littérature reconnue?}

Cela étant, et puisque, tel que j'ai cherché à le souligner au début de cet article, la communauté franco-albertaine du XXI ${ }^{e}$ siècle est moins préoccupée par sa survie que par son épanouissement et sa reconnaissance auprès d'autrui, je m'intéresserai dans la suite à découvrir ce qu'il en est de ses efforts pour rendre visible sa littérature. Dans une conférence présentée au Campus Saint-Jean le 27 septembre 2007, l'écrivain et critique littéraire Pierre Nepveu prétendait que si ce que le Québec connaissait des littératures des "autres francophonies "du pays se limitait à celles des régions limitrophes du Québec, c'était pour des raisons liées certes à la distance, mais encore plus, au manque de diffusion : "Nous n'en entendons presque jamais parler. [...] Le manque de moyens financiers empêche aussi ces communautés-là de sortir $^{23}$ ". S’il y a sans doute du vrai dans ce constat, on ne saurait nier que les écrivains francophones du Far Ouest sont moins inconnus dans des parties du Canada encore plus lointaines que le Québec : en Acadie, par exemple. Or, mon intention n'est pas d'encore revenir sur la question des rapports entre les francophones de l'Alberta et du Québec, mais plutôt d'affirmer que les universitaires canadiens œuvrant en dehors du Québec ont fait de leur mieux pour accroître la reconnaissance des écrivains franco-albertains et ce, depuis belle lurette. Pour s'en convaincre, on n'aurait qu'à consulter les travaux de chercheurs tels Roger Motut, Jules Tessier, Estelle Dansereau, Paul Dubé, Lise GabouryDiallo, moi-même et j'en passe. Seulement, depuis que l'on présente des entretiens ou des communications savantes à leur sujet, qu'ils soient nés en Alberta ou qu'ils s'y sont établis pour

23 Cité dans Le Franco, 5 octobre 2007, p. 5. 
vivre et travailler, qu'ils écrivent en français, en anglais ou dans les deux langues et ce, dans un même ouvrage ou bien dans deux ouvrages différents, on a eu tendance à produire des études portant sur un auteur, ou parfois, sur deux ou trois. À défaut d'une histoire littéraire ayant le potentiel de présenter une perspective relativement large du Far Ouest francophone, je me tournerai vers un article écrit pour un ouvrage auquel aurait accès tout Franco-Canadien hors-Québec : L'Encyclopédie canadienne.

Dans «Littérature d'expression française dans l'Ouest canadien ", publié en 2000, Ismène Toussaint, une francophone originaire de la Bretagne, qui, après avoir vécu neuf ans au Manitoba, s'est installée en 1998 au Québec, observe que compte tenu des obstacles auxquels ont fait face la minorité francophone de l'Ouest, la survie de sa littérature "relève bel et bien du miracle $^{24}$ ». Née sur les bords de la rivière Rouge au Manitoba, la plus grande part de la littérature éponyme se manifeste toujours dans cette province-là, affirme l'auteure, avant d'annoncer son intention de privilégier la production franco-manitobaine tout en citant les noms et les ouvrages des auteurs les plus marquants des « autres " provinces de la région - la Saskatchewan, l'Alberta, la Colombie-Britannique et les Territoires du Nord-Ouest. Il s'avère que parmi les six périodes historiques ${ }^{25}$ servant à jalonner l'évolution de la littérature dans l'Ouest, quatre sont marquées par la contribution de six écrivains « de » l'Alberta. L'Alberta est la province natale de quatre d'entre eux - Marguerite-A. Primeau, Jean Pariseau, Guy Pariseau et Nancy Huston -, et la province d'adoption des deux autres - Georges Bugnet et Marie-Anna Roy. Quant à leur statut d'écrivain, Bugnet, Primeau et Huston jouent un rôle considéré comme majeur pendant l'une des périodes productives, tandis que chacun des frères Pariseau a joué un rôle

\footnotetext{
24 Ismène Toussaint, "La Littérature d'expression française de l'Ouest ", L'Encyclopédie canadienne, Historica-Dominion, 2013. (Le texte date de 2000.); [en ligne] http://www.thecanadianencyclopedia.com, consulté le 26 février 2013.

25 L'article rend compte de cette production littéraire selon six périodes : [1] 1700 - 1900; [2] 1900 - 1945; [3] 1945 - 1960; [4] 1960 - 1975; [5] 1975 -1990 siècle; et [6] 1990 jusqu'au début du millénaire.
} 
qui, bien que considéré comme digne de mention, fut tout sauf éclipsé par la figure majeure de leur époque ${ }^{26}$. Curieusement, pour la période allant de 1945 jusqu'en 1960, la section intitulée "Un désert fleuri de roses " accorde dix-neuf lignes à Gabrielle Roy, ce qui ne doit étonner personne, mais c'est pour ensuite consacrer douze lignes à sa sœur aînée qui, " du fond de son Alberta d'adoption ", a signé des " écrits pionniers de la terre " tandis que le genre était passé de mode.

Bugnet, né en France en 1879, mais établi en Alberta de 1905 jusqu'à son décès en 1981, y est identifié comme l'un de trois auteurs " Maîtres " de l'époque des "Pionniers de la terre et de la plume (1900-1945) ${ }^{27}$ ». Primeau, francophone originaire de l'Alberta où elle est née en 1914, mais établie à Vancouver de 1954 jusqu'à son décès en 2011, est l'un des deux écrivains ${ }^{28}$ ayant marqué la période allant de 1960 jusqu'en 1975, grâce à qui « la littérature de l'Ouest esquisse enfin un timide réveil dans les années 60 ». Huston, née en 1953 à Calgary, mais établie à Paris depuis 1973, est considérée comme l'un des «pionniers du nouveau millénaire »: " [a] u royaume du roman, écrit Toussaint, règne incontestablement, depuis quelques années, l'Albertaine Nancy Huston ". À l'égard des autres écrivains francophones contemporains « de » l'Alberta, c'est-à-dire qui y sont nés ou bien qui y vivent et travaillent ou qui y ont vécu et travaillé, L'Encyclopédie canadienne n'indique rien. Et pourtant, en lisant Toussaint qui affirme, vers la fin de son article, qu'au début du $\mathrm{XXI}^{\mathrm{e}}$ siècle, " [p]ar-delà des essais universitaires secs et sans âme,

$26 \quad$ Pour la période allant de 1975 jusqu'en 1990, le Franco-Manitobain Roger Léveillé se distingue comme le chef de file d'une poésie nouvelle de la révolte, dans le "sillage " de qui écrit " toute une farandole de poètes turbulents et tapageurs, [dont] Jean Pariseau, Guy Pariseau, etc. ».

27 Les deux autres écrivains phares sont Maurice Constantin-Weyer et Jean Féron. Le premier, un Français qui a séjourné au Manitoba de 1904 jusqu’en 1914, période pendant laquelle il a pris la nationalité canadienne, a été le récipiendaire du Prix Goncourt en 1928 pour son roman Un homme se penche sur son passé, basé sur ses expériences manitobaines. Le second, un Québécois établi dans une ferme en Saskatchewan, a signé, entre autres, La Métisse en 1923.

28 L'autre est Roger Léveillé du Manitoba, dont l'écriture est aussi innovante que celle de Primeau est " classique». 
des analyses linguistiques indigestes et des études critiques peu professionnelles, la création se porte plutôt bien dans l'Ouest et que la relève semble solidement assurée ", je me permets d'être d'un autre avis en ce qui concerne les études des chercheurs universitaires, mais je n'hésite nullement à confirmer que l'optimisme exprimé à l'égard de la création littéraire "dans l'Ouest » s'étend à celle du «Far Ouest». Depuis l'an 2000, non moins de treize artistes littéraires ont publié un texte ou un ouvrage ou bien performé ou lu en public des textes courts ou extraits de texte : ils s'appellent (en ordre alphabétique) Guy Armel Bayegnak $^{29}$, Pascale Bourassa ${ }^{30}$, Paulette Dubé ${ }^{31}$, Tchitala $\mathrm{Kamba}^{32}$, Eileen Lohka ${ }^{33}$, Nadine Mackenzie ${ }^{34}$, Denise Ouellette-

29 Guy Armel Bayegnak, camerounais d'origine, a publié deux romans : Coeur de lionne, Saint-Boniface, Éditions du Blé, 2011, 270 p. et Le Plancher se dérobe, Saint-Boniface, Éditions du Blé, 2012, 184 p.

30 La Québécoise Pascale Bourassa enseigne le français à Fahler en Alberta depuis 2005. Soit dit en passant, en 2012-2013, elle a pris un congé sabbatique de son poste albertain et se retrouve au Québec. Elle a publié deux romans : Le Puits, Saint-Sauveur-des-Monts (Québec), La Grenouillère, 2009, 144 p. et À l'ouest, Saint-Sauveur-des-Monts (Québec), La Grenouillère, 2012, 235 p.

31 Paulette Dubé, née et élevée en français en Alberta, est l'auteure primée de nombreux recueils de poésie écrits en anglais et d'un roman, Talon, Edmonton, NeWest, 2002, 215 p., écrit principalement en anglais, mais avec un grand nombre de passages en français.

32 Tchitala Kamba, originaire du Congo, a publié un ouvrage de fiction jusqu'ici, L'Exilée de Makelele, Saint-Boniface, Éditions des Plaines, 2007, 64

33 p.

Eileen Lohka, originaire de l'Île Maurice, est l'auteure de Miettes et morceaux, Quatre Bornes, Île Maurice, Bartholdi, 2005, 144 p., et de C'était écrit, Ottawa, L'Interligne, 2010, 104 p.

34 Nadezhda (Nadine) Mackenzie est d'origine suisse alémanique et russe, mais est établie au Canada (à Calgary actuellement) depuis 1974. Elle est publiée depuis 1978, aux éditions des Plaines, du blé et de la Nouvelle Plume et jusqu'ici, a signé dix-sept ouvrages de fiction. La majorité des titres visent des jeunes lecteurs, mais sa bibliographie inclut aussi cinq romans, une nouvelle et un récit destinés à des lecteurs adultes. 
Berkhout $^{35}$, Danièle Petit ${ }^{36}$, Claudine Potvin ${ }^{37}$, Pierrette Requier ${ }^{38}$, Josée Thibeault ${ }^{39}$, Jocelyne Verret-Chiasson ${ }^{40}$ et Gisèle Villeneuve ${ }^{41}$.

L'article de Toussaint a été écrit en 2000, mais demeure tel quel dans l'édition de 2013 de L'Encyclopédie canadienne. C'est dommage parce que, non seulement un ouvrage censé être un ouvrage de référence national réduit-il la production littéraire franco-albertaine contemporaine à l'œuvre d'une seule écrivaine, mais de plus, cette écrivaine a suivi un parcours qui la rend inconsciente des réalités des francophones minoritaires de l'Alberta. Élevée dans la langue anglaise, à Calgary jusqu'à l'âge de six ans et ensuite, aux États-Unis, Nancy Huston a suivi des cours de français, puis à l'âge de vingt ans, elle s'est établie à Paris où, au fur et à mesure de nombreux essais et ouvrages de fiction, elle est devenue l'écrivaine d'expression française ${ }^{42}$ qu'on connaît

35 Originaire de l'Ontario, Denise Ouellette-Berkhout est établie à Calgary depuis le début des années 1980. Jusqu'ici, elle a signé cinq romans, tous publiés aux éditions des Plaines à Saint-Boniface, MB : Bonjour garde, 1994, 222 p.; Quand jaurai retrouvé mon fils, 1998, 220 p.; Le Golé, 2002, 128 p.; Le Diamant du Jood, 2007, 160 p.; et Collé à la peau, 2012, 157 p.

36 Danièle Petit, originaire de la région Touraine en France, mais établie à Edmonton depuis 1971, est l'auteure de poèmes parus dans certaines revues, dont Confluence (1999), et de textes diffusés par Radio-Canada.

37 Québécoise d'origine, mais professeure à l'Université de l'Alberta de 1986 jusqu'en 2010, Claudine Potvin a publié plusieurs études universitaires, mais aussi deux ouvrages littéraires : Détails : contes autobiographiques, 1993, $97 \mathrm{p}$. et Pornographies, 2002, 130 p., tous deux aux éditions de L'instant même, au Québec.

38 Il s'agit de l'auteure de Details from the Edge of the Village, Calgary, Frontenac House, 2009, 109 p.

39 Metteure en scène, Josée Thibeault n'est pas encore publiée, mais a fait la lecture publique de nombreuses compositions siennes.

40 Elle a signé, entre autres, deux recueils bilingues de poésie publiés à compte d'auteur : Forêts et océans/Of Trees and Sea, 1994, et Gens d'ici, gens d'ailleurs/ People from Here and Afar, 1996; une pièce de théâtre, Voulez-vous danser?, Régina, Éditions de la nouvelle plume, 1996, 99 p.; et un roman, J'attendrai, Saint-Boniface, éditions des Plaines, 2003, 126 p.

41 Écrivaine, traductrice, correctrice et scénariste, entre autres, Gisèle Villeneuve, originaire de Montréal, mais établie à Calgary depuis 1976, est l'auteure de plusieurs textes dramatiques et radiophoniques et aussi, de deux romans, Rumeurs de la Haute Maison, Montréal, Québec/Amérique, 1987, et Visiting Elizabeth, Montréal, XYZ, 2004.

$42 \mathrm{Si}$, depuis Cantique des plaines, elle est aussi une romancière d'expression anglaise, en tant qu'essayiste, Huston écrit uniquement en français. 
aujourd'hui. Publiée principalement aux Actes Sud en France et chez Leméac au Québec, la coqueluche des média dans son pays d'adoption, bien reçue au Québec, saurait-elle seulement imaginer la minorisation des francophones de l'Alberta ou bien les conditions dans lesquelles les artistes littéraires francophones de sa province natale cherchent à percer? Et pourtant, il faudrait bien admettre que sa renommée d'écrivaine a des retombées sans doute positives pour le statut de la littérature d'expression française associée à l'Alberta - je pense notamment à son roman Cantique des plaines, le premier ouvrage de fiction que l'auteure dit avoir écrit d'abord en anglais avant de le réécrire en français et ce, afin de traiter de sa province natale. Il conviendrait de souligner, toutefois, que la version française de l'ouvrage contribue à renforcer la tendance à limiter les questions touchant la francophonie canadienne à la seule province du Québec, car si le texte parle principalement de l'Alberta, le « je " écrivant, lui, se trouve à Montréal ${ }^{43}$.

\section{L'Alberta francophone par sa littérature à l'époque du Canada français}

En 1915, Louis-Arthur Prud'homme soutenait auprès de ses collègues de la Société Royale du Canada que "la vague des milliers de colons qui se déverse à tous les ans sur nos prairies a déterminé la fièvre d'une spéculation intense qui étouffe le libre essor de nos jeunes talents littéraires. [... La] chronique, pressée de noter au passage les événements du jour, tient le premier rang $^{44}$ ». Cela pourrait bien être, mais il n'en est pas moins vrai

43 Voir à ce sujet Pamela V. Sing, «Écrire l'absence : Montréal et l'Alberta chez Marguerite-A. Primeau et Nancy Huston ", University of Toronto Quarterly, vol. 70, no 3, été 2001, p. 737-751 et Pamela V. Sing, "Stratégies de spatialisation et effets d'identification ou de distanciation dans Cantique des plaines ", dans Marta Dvorák et Jane Koustas (dir.), Vision/division, l'œuvre de Nancy Huston, Ottawa, Presses de l'Université d'Ottawa, 2004, p. 63-74.

44 "La littérature française au Nord-Ouest ", Mémoires de la Société Royale du Canada, Section I, Série III, vol. IX, décembre 1915, p. 247. [Texte lu à la réunion de mai 1915]. Prud'homme enjoint ses collègues à «tenir par la main [leur] Benjamin de l'Ouest, afin de mieux favoriser les premiers battements de ses ailes [en lui permettant de leur] chanter, sur un modeste pipeau, les 
que l'élite franco-albertaine de l'époque n'était pas en mesure d'encourager les activités d'ordre littéraire.

Georges Bugnet, qui a mis l'Alberta francophone sur la carte littéraire du pays ${ }^{45}$, écrivait dans une solitude et un isolement presque absolus. "Presque ", dis-je, car il avait pour correspondant un certain nombre de figures du milieu littéraire au Québec, dont Camille Roy, à qui il envoyait des textes et auprès de qui il cherchait une confirmation de la valeur du fruit de son labeur. L'extrait suivant d'une lettre datée du 12 janvier 1924 témoigne de l'émotion de l'écrivain lorsque le critique littéraire lui a écrit après avoir lu le manuscrit de sa nouvelle, "Le Pin du Muskeg »:

Cher Monsieur l'abbé,

Votre lettre m'a fait un très grand plaisir. C'est la première fois que dans ce lointain Québec, je trouve une âme qui sintéresse non seulement à mes écrits mais aussi à moi-même. La surprise m'a fait monter les larmes aux yeux ${ }^{46}$.

En 1927, Bugnet écrivait à son correspondant qu'» Edmonton [était] fort mal meublé en fait de littérature canadienne-française $^{47}$ » et en 1930, il se lamentait d'être " une pauvre molécule humaine [...] isolée de tout centre intellectuel ${ }^{48} »$. Ce néanmoins, grâce à la qualité de son écriture ${ }^{49}$, puisqu'il écrivait à l'époque du Canada français et que son œuvre révélait une région jusqu'alors peu connue du Canada français, des historiens littéraires allant de Camille Roy (1939) à Gérard Bessette (1968) en passant par Berthelot Brunet (1946) et Gérard Tougas (1960)

travaux, les luttes et les espérances de [leurs] frères de là-bas. » (p. 248)

Le Lys de sang, 1923; Nipsya, 1924; La Forêt, 1935, Journal 1954-1971, 1984; des essais : Siraf, 1934 et Canadiana, 1941 et de la poésie : Les Voix de la solitude, 1938. Albertaines: anthologie d'œuvres courtes en prose, 1990.

46 Archives du Séminaire de Québec, FO Camille Roy, Carton 89, n 2, Georges Bugnet à Camille Roy, le 12 janvier 1924.

47 Archives du Séminaire de Québec, FO Camille Roy, Carton 89, n 9, Georges Bugnet à Camille Roy, le 27 janvier 1927.

48 Archives du Séminaire de Québec, FO Camille Roy, Carton 89, $\mathrm{n}^{\circ} 15$, Georges Bugnet à Camille Roy, le 19 décembre 1930.

49 Dans son «L'Ouest canadien et sa littérature », essai publié en 1986, Annette Saint-Pierre le surnommait le " poète des roses » en avançant qu'avec Maurice Constantin-Weyer et Jean Féron, il avait donné à la littérature éponyme ses lettres de noblesse (Revue d'histoire littéraire du Québec et du Canada français, $\mathrm{n}^{\circ} 12$, p. 171-200). 
ont consacré un nombre considérable de pages à son œuvre, ce qui a valu à lui et à ses ouvrages principaux et ergo, le Far Ouest francophone, d'être reconnus dans l'Histoire de la littérature québécoise de Michel Biron, François Dumont et Élisabeth Nardout-Lafarge, publiée en 2007. (Soit dit en passant, ce dernier ouvrage fait référence au milieu d'édition de l'Ouest, c'est-à-dire du Manitoba, mais hormis Bugnet, passe sous silence tous les écrivains francophones ayant écrit et publié à l'ouest de l'Ontario.) Par ailleurs, non seulement plusieurs de ses textes sont-ils connus dans une version anglaise - son roman, Nipsya, a été traduit en anglais en $1929^{50}$, et son roman, La Forêt, en $1976^{51}$, par exemple -, mais de plus, des dizaines d'études, d'articles scientifiques, de mémoires et de thèses écrits à travers le Canada ont eu pour objet un aspect ou un autre de son œuvre ${ }^{52}$.

Pendant le premier tiers du XX $\mathrm{XX}^{\mathrm{e}}$ siècle, d'autres francophones albertains écrivaient aussi et, Bugnet, soucieux de faire reconnaître l'existence d'une littérature " canadienne ", déplorait leur oubli. La lecture du manuel de littérature de Camille Roy, notamment, l'a poussé à correspondre avec l'auteur pour lui dire qu'à son humble avis, les "rares " bonnes plumes de l'Ouest "français " appartenaient à lui-même, certes, mais aussi à Donatien Frémont et aux Pères Morice, Petitot et Duchaussois, et que ledit manuel devait les inclure ${ }^{53}$. À ces noms-là, il faudrait ajouter ceux des auteurs des textes courts - nouvelles, poèmes, contes - publiés dans les journaux d'expression française de la province. Hormis une place dans le Dictionnaire des Artistes et Auteurs francophones de l'Ouest canadien de Gamila Morcos, toutefois, le rôle qu'ils ont joué dans la construction de l'image de la francophonie albertaine a été peu reconnu. Quant à l'image de l'Alberta francophone véhiculée dans l'œuvre de Bugnet, je

\footnotetext{
50 Georges Bugnet, Nipsya, trad. par Constance Davies Woodrow, New York, L. Carrier, 1929, 285 p.

51 Georges Bugnet, The Forest, trad. par David Carpenter, Harvest House, 1976, $172 \mathrm{p}$.

52 Pour des informations d'ordre bibliographique, voir Cahiers franco-canadiens de l'Ouest, vol. $11, \mathrm{n}^{\text {os }} 1$ et $2,1999$.

53 Archives du Séminaire de Québec, FO Camille Roy, Carton 89, $\mathrm{n}^{\circ} 11$, Georges Bugnet à Camille Roy, le 20 février 1930.
} 
me limiterai ici à mentionner que Monique Bosco a traité de son œuvre dans sa thèse de doctorat portant sur l'isolement dans la littérature canadienne-française ${ }^{54}$.

\section{L'Alberta francophone par sa littérature, 1960-1999}

Entre 1960, année associée au début de la Révolution tranquille, et 1996, le Far Ouest francophone a continué à travailler à assurer sa survie. Sa littérature d'expression française s'est alors manifestée principalement au travers de la seule ouvre de Marguerite-A. Primeau, franco-albertaine d'origine, je le rappelle, mais établie à Vancouver dès 1954. Elle a fait paraître son premier roman, publié à Montréal, en $1960^{55}$, puis, entre 1983 et 1996, avec l'une des deux maisons d'édition francomanitobaines, deux autres romans, dont l'un s'est mérité le prix littéraire Champlain, et deux recueils de nouvelles ${ }^{56}$. En 2005, finalement, elle a écrit un dernier récit pour un collectif destiné à commémorer en français le centenaire de l'Alberta ${ }^{57}$. Tous ses textes font connaître la francophonie albertaine.

En Colombie-Britannique, Radio-Canada, deux journaux franco-colombiens, Le Soleil de Colombie (fondé en 1968) et L'Express du Pacifique (fondé en 1998), et, entre 2004 et 2007, l'Association des écrivains francophones de la ColombieBritannique, avec son siège social dans l'île de Vancouver, ont contribué à faire reconnaître l'œuvre de celle que la francophonie franco-colombienne considère comme la doyenne de ses écri-

54 Monique Bosco, L'Isolement dans le roman canadien-français, thèse de doctorat, Université de Montréal, 1953, 205 p.

55 Marguerite-A. Primeau, Dans le muskeg, Montréal, Fides, 222 p. (réédité aux éditions des Plaines en 2005).

56 Marguerite-A. Primeau, Maurice Dufault, sous-directeur, Saint-Boniface, Éditions des Plaines, 1983, 200 p.; Sauvage Sauvageon, Saint-Boniface, Éditions des Plaines, 1984, 159 p., ouvrage auquel on a décerné le prix Champlain en 1984 (réédité en 2004); Le Totem, Saint-Boniface, Éditions des Plaines, 1988, 154 p.; et Ol' Man, Ol' Dog et l'enfant et autres nouvelles, Saint-Boniface, Éditions du blé, 1996, 84 p. (réédité, revu et augmenté en 2004).

57 Marguerite-A. Primeau, "Saint-Paul-des-Métis », dans Estelle Dansereau, Pamela V. Sing, Eileen Lohka et Paul Dubé (dir.), Alberta, village sans mur(s), Saint-Boniface, Presses Universitaires de Saint-Boniface, p. 7-14. 
vains. En Alberta, ont mis la main à la pâte trois instances institutionnelles - Radio-Canada, La Revue littéraire de l'Alberta qui a existé le temps de publier cinq ou six numéros, soit de 1982 à 1986, et l'hebdomadaire Le Franco - et des universitaires francophones et anglophones ${ }^{58}$. En 1996, deux études approfondies de son œuvre avaient paru. Déterminée à accroître la visibilité de l'œuvre de Primeau, je me suis mise à rédiger une vingtaine d'études sur différents aspects de ses textes, lesquelles ont été publiées dans des revues scientifiques ou livres édités principalement dans l'Ouest francophone ou dans une autre partie du Canada francophone hors-Québec, mais aussi au Canada anglais, au Québec ou aux États-Unis. Vers la même époque, Margaret Wilson s'est mise à travailler à la traduction en anglais de deux ouvrages de Primeau ${ }^{59}$ et, ensuite, dans les années 2000, tandis que j'ai assuré la réédition de trois de ses ouvrages, un quatrième a été traduit en anglais par Maureen Ranson ${ }^{60}$.

Primeau s'avère la seule écrivaine franco-albertaine d'expression française de la seconde moitié du XXe siècle, mais vers la fin des années 1980, deux autres écrivaines franco-albertaines, Marie Moser et Jacqueline Dumas, ont publié chacune un roman traitant du Far Ouest francophone, mais en anglais ${ }^{61}$. Élèves à l'époque où l'Alberta autorisait un enseignement en français uniquement pendant les deux premières années à l'élémentaire, après quoi les écoliers francophones subissaient une assimilation à l'anglais progressivement plus complète, Moser et Dumas ne maîtrisent pas suffisamment leur langue maternelle pour pouvoir

58 Dont, entre autres, Jules Tessier de l'Université d'Ottawa, Paul Dubé, de l'Université de l'Alberta, Estelle Dansereau de l'Université de Calgary, Jimmy Thibeault de l'Université de Moncton (actuellement, de celle de SainteAnne), E.D. Blodgett de l'Université de l'Alberta et George Melnyk de l'Université de Calgary.

59 Marguerite-A. Primeau, Savage Rose, trad. par Margaret M. Wilson, Victoria, Ekstasis Editions, 1999, 146 p. et Marguerite-A. Primeau, The Totem, trad. par Margaret M. Wilson, Victoria, Ekstasis Editions, 2002, 128 p.

60 Marguerite-A. Primeau, Maurice Dufault, Vice-Principal, trad. par Maureen Ranson, Calgary, Presses Universitaires de Calgary, 2006, 222 p.

61 Marie Moser, Counterpoint, Toronto, Irwin Publishing, 1987, 166 p. et Jacqueline Dumas, Madeleine \& the Angel, Saskatoon, Fifth House Publishers, $1989,188 \mathrm{p}$. 
en faire leur langue d'écriture. Seul l'ouvrage de Moser a été traduit en français, mais les deux romans ont été primés. La version originale et la traduction en anglais de celui de Moser, qui lui a valu le prix du New Alberta Novel Competition de 1986, sont épuisées depuis au moins une décennie. Quant à celui de Dumas, pour lequel l'auteure a été le récipiendaire du prix George Bugnet pour le meilleur premier roman albertain en 1989, il a connu une suite sous la forme d'une pièce de théâtre intitulée Secrets, qui met en scène les deux sœurs du roman, quarante ans après le décès de leur mère.

Il en ressort que pendant une cinquantaine d'années, grâce aux ouvrages lus, primés, célébrés et étudiés, de trois écrivaines franco-albertaines, le Far Ouest francophone a été reconnu par des lecteurs dans au moins trois des communautés suivantes : le Canada francophone at large, en particulier en Alberta et en Colombie-Britannique, le Canada anglais at large, et, du moins pour certains ouvrages de Primeau, les États-Unis.

Or, en Alberta francophone, comme partout ailleurs dans le pays, fort probablement, les manifestations "festivalesques " populaires, dont des cabanes à sucre ou des carnavals régionaux, attirent plus de monde et par conséquent, ont plus de valeur symbolique pour la communauté, que la littérature. Selon son rapport annuel de 2011-2012, sous la rubrique "vie culturelle", définie comme " préservation du patrimoine, arts et culture, promotion et visibilité de la francophonie, etc. ", le Secrétariat francophone de l'Alberta a appuyé neuf festivals ou manifestations de la sorte, dont le Carnaval de Saint-Isidore qui, selon le géographe Dean Louder, est le " coin le plus vivant " de la francophonie actuelle ${ }^{62}$. L'ensemble de ces manifestations ont effectivement attiré plus de 30,000 spectateurs $^{63}$. Qu'elles contri-

62 Lors d'une conversation tenue à Montréal le 10 mai 2010. Soit dit en passant, l'un de mes étudiants avait assisté au Carnaval de St-Isidore en 2012, et à son retour à Edmonton, m'a communiqué son enthousiasme et admiration, affirmant que des gens " de partout, même de la Colombie-Britannique " avaient entendu parler du Carnaval de St-Isidore.

63 L'appui du Secrétariat francophone est rendu possible dans le cadre d'une entente entre les gouvernements fédéral et provincial. Si, entre 2001 et 2006, le Secrétariat francophone n'a pas appuyé de festival, il en a appuyé cinq en 
buent à accroître la visibilité de la francophonie albertaine ne fait pas de doute, mais il n'en demeure pas moins que par de telles activités, les signes visibles de l'identité culturelle des Francoalbertains continuent de résider dans la tourtière, la tarte au sucre, la musique folklorique variablement "modernisée " et l'artisanat ${ }^{64}$. Par ailleurs, il faut bien reconnaître que le succès de telles manifestations culturelles participe du dynamisme actuel de la communauté franco-albertaine et que ce dynamisme s'est accompagné non seulement d'une production littéraire de plus en plus foisonnante, mais aussi d'efforts pour la rendre visible et pour la célébrer.

\section{Dynamisme communautaire et foisonnement littéraire au $\mathrm{XXI}^{\mathrm{e}}$ siècle}

Depuis les années 1970, les écrivains francophones de l'Alberta n'ont plus à chercher à être publiés au Québec, car la communauté franco-manitobaine s'est dotée de deux maisons d'édition, le blé, fondé en 1974, et les Plaines, fondées en 1979, qui toutes deux ouvrent leurs portes aux écrivains de l'Ouest entier. En revanche, le prix littéraire Rue-Deschambault, créé en 1989, parrainé par le ministère de la Culture, du Patrimoine et du Tourisme de la province du Manitoba, et géré par le Manitoba’s Book Guild, est réservé aux écrivains manitobains. L'Alberta francophone n'a ni maison d'édition ni prix littéraire, mais en 2001, le Regroupement artistique francophone de l'Alberta (RAFA), l'un des treize organismes provinciaux et territoriaux membres de la FCCF, a été fondé65. Sa mission officielle étant " de regrouper l'ensemble des artistes, des intervenants et des organismes culturels et

2006-07 et 2007-08, après quoi le nombre annuel a augmenté jusqu’à 9 à partir de 2011.

64 La même année, il a aussi appuyé les célébrations du 150e anniversaire de la ville de Saint Albert, le projet du Conseil de développement économique de l'Alberta, qui, sous la forme d'une visitée guidée, visait à faire la promotion des racines francophones de la ville de Calgary et, en fin de compte, la mise en valeur de l'histoire francophone de l'Alberta aux Archives provinciales de l'Alberta.

65 La francophonie albertaine est la dernière de l'Ouest à avoir fourni à ses artistes culturelles une telle institution d'appui. 
artistiques d'expression française pour assurer le développement et l'épanouissement de toutes les formes d'expressions artistiques en Alberta ${ }^{66}$ ", l'organisme publie un bulletin électronique hebdomadaire intitulé Coup d'oil auquel on peut s'abonner gratuitement ${ }^{67}$ pour $s^{\prime}$ informer de ce qui se passe dans les sept secteurs des arts et de la culture franco-albertaine qu'il appuie : chanson/musique, théâtre, danse, événements en province, arts littéraires, arts médiatiques et art visuel. De plus, son site Web donne accès à son portail littéraire, Alinéa, ayant pour mission de

promouvoir la littérature des écrivains albertains d'expression française de toute origine culturelle. Il comprend les notices bibliographiques, les bibliographies et les dossiers de presse des écrivains d'expression française natifs de l'Alberta, immigrés, émigrés ou albertains d'adoption, auteurs de romans, nouvelles, contes, poésie, théâtre, essais ou études, biographies ou littérature jeunesse. Il affiche également les dates des activités liées au secteur littéraire prévues pour l'année en cours ${ }^{68}$.

Parmi les différentes façons dont le RAFA contribue au dynamisme du milieu littéraire, je retiens ici l'organisation d'événements littéraires, dont des soirées de lecture publique, la création de partenariats et l'établissement de ponts avec d'autres organismes, pour que les écrivains aient des occasions de se faire

66 Sur son site Web, on peut lire ceci : « Le Regroupement artistique francophone de l'Alberta (RAFA) est un organisme provincial de services. Sa mission est de regrouper l'ensemble des artistes, des intervenants et des organismes culturels et artistiques d'expression française pour assurer le développement et l'épanouissement de toutes les formes d'expressions artistiques en Alberta. Fondé en 2002 par la communauté artistique, le RAFA détient le statut officiel d'organisme porte-parole des arts et de la culture d'expression française en Alberta. Son mandat est de regrouper, de développer et de faire progresser l'ensemble des disciplines artistiques afin d'assurer dans toute la province, le développement progressif et continu de l'industrie culturelle et artistique.

Depuis avril 2005, le RAFA est reconnu officiellement par le gouvernement de l'Alberta à titre d'organisme parapluie des arts et de la culture francophone. Le Regroupement a été intégré au rang du PASO (Provincial Arts Service Organization). Ce qui donne au RAFA un plus grand poids politique, des moyens de développement et des capacités plus grandes de mise en réseau avec les divers partenaires albertains œuvrant dans l'industrie culturelle et artistique. "

67 [en ligne] http://www.rafa-alberta.ca/coupdoeil, consulté le 26 février 2013.

68 [en ligne] http://www.rafa-alberta.ca/alinea/, consulté le 26 février 2013. 
connaître dans d'autres milieux et ce, dans la province aussi bien que hors-Alberta. En cherchant à mettre les écrivains en contact entre eux et avec les artistes d'autres champs, d'autres milieux, d'autres communautés, il multiplie les interactions et contribue à créer une énergie et une reconnaissance mutuelle locale qui conduit à des partenariats et des projets stimulants et prometteurs.

Par ailleurs, Liaison, une " revue d'information, d'opinion et de création artistique " jadis consacrée à la création artistique franco-ontarienne, mais s'intéressant depuis 2005 à la production artistique pancanadienne, contribue, elle aussi, à faire reconnaître la littérature francophone de l'Alberta. Dans son premier numéro pancanadien (il s'agit du $\mathrm{n}^{\circ} 129$ ), le dossier sur l'Ouest, confié à Paul Dubé, était composé de vingt-et-une pages. On a été six à y avoir contribué des pages traitant des romans de l'Ouest, de sa poésie, de sa littérature de jeunesse, de l'édition, de ses écrivaines francophones qui écrivent en anglais et de Marguerite-A. Primeau. Huit ans plus tard, dans le numéro du printemps 2013 (il s'agit du $n^{\circ}$ 159), le Far Ouest fait une deuxième parution dans la revue. L'article de Paul Savoie, intitulé "La relève littéraire ", parle de trois auteurs et leurs ouvrages, dont Le plancher se dérobe de Guy Armel Bayegnak, qui, de surcroît, a été l'un des ouvrages finalistes du concours littéraire Prix des lecteurs Radio-Canada 2013.

Force nous est de conclure avec l'écrivaine Paulette Dubé que " quelque chose bouge » dans le domaine :

Mes écrits (en prose) [livrent des observations au sujet de] la vie historique d'un petit groupe franco-albertain [qui vit] dans un petit coin de la province. Je pense que plusieurs personnes savaient qu'on était là, mais ils ne savaient pas pourquoi on était là [et ne connaissaient pas non plus] notre histoire, nos traditions de guérisseurs. Il y [a eu] les œuvres de Georges Bugnet, bien sûr et, de Marguerite Primeau [...], mais on ne saurait dire qu'à deux, il y a eu foule. Au cours des 40 dernières années, [on assiste à la résurgence] de la langue [que] certains disaient déjà $[. .$.$] vaincue. [Elle] est de retour comme un ruisseau dans un$ paysage aride. Nous sommes moins de 10000 personnes à parler cette langue, c'est une langue précieuse. Elle brosse un tableau francophone 
qui est nettement albertain avec ses chevaux, le grand vent du nord et ses taches d'étoiles, d'hivers rigoureux et de prières ${ }^{69}$.

Non seulement des lecteurs des autres francophonies du Canada commencent-ils à (re)connaître celle du Far Ouest, mais aussi le Canada "anglais » semble se réveiller enfin à ses francophones du Far Ouest, une communauté depuis longtemps méconnue. Que les artistes littéraires francophones de l'Alberta révèlent différents aspects de la communauté au sein de laquelle ils pratiquent leur art dans des ouvrages écrits en français ou en anglais, leurs mots participent de la vitalité de cette communauté, en témoignent et en la faisant exister dans l'imaginaire de leurs lecteurs, ils contribuent à braquer sur elle les feux d'un nouvel éclairage.

69 Dans une communication personnelle datant du 7 avril 2010. 


\section{Bibliographie}

ACFA, http://www.acfa.ab.ca/Documents/Communiques2010/20130226_ Membership-de-l-ACFA-Vers-une-vision-commune.pdf, consulté le 26 février 2013.

Bayegnak, Guy Armel, Cour de lionne, Saint-Boniface, Éditions du Blé, 2011, 270 p.

Bayegnak, Guy Armel, Le Plancher se dérobe, Saint-Boniface, Éditions du Blé, 2012, 184 p.

Behiels, Michael D., La Francophonie canadienne : renouveau constitutionnel et gouvernance scolaire, Ottawa, Presses de l'Université d'Ottawa, 2005, $462 \mathrm{p}$.

Bosco, Monique, L'Isolement dans le roman canadien-français, thèse de doctorat, Université de Montréal, 1953, 205 p.

Bourassa, Pascale, À l'ouest, Saint-Sauveur-des-Monts (Québec), La Grenouillère, 2012, 235 p.

Bourassa, Pascale, Le Puits, Saint-Sauveur-des-Monts (Québec), La Grenouillère, 2009, 144 p.

Bugnet, Georges, Archives du Séminaire de Québec, FO Camille Roy, Carton 89, $\mathrm{n}^{\text {os }} 2,9,11,15$, Georges Bugnet à Camille Roy, 12 janvier 1924, 27 janvier 1927, 20 février 1930, 19 décembre 1930.

Bugnet, Georges, The Forest, trad. par David Carpenter, Harvest House, 1976, $172 \mathrm{p}$.

Bugnet, Georges, Nipsya, trad. par Constance Davies Woodrow, New York, L. Carrier, 1929, 285 p.

Cambron, Micheline, "L'Enchevêtrement des histoires littéraires dans la francophonie d'Amérique vu à travers le renouvellement épistémologique de l'histoire littéraire ", Francophonies d'Amérique, no 26, 2008, p. 345-355.

Castonguay, Charles, "Getting the Facts Straight on French Reflections Following the 1996 Census ", Inroads, no 8, 1999, p. 57-77.

Dubé, Paulette, Talon, Edmonton, NeWest, 2002, 215 p.

Dumas, Jacqueline, Madeleine \& the Angel, Saskatoon, Fifth House Publishers, 1989, $188 \mathrm{p}$.

Ferron, Jacques, "La Vache morte au canyon ", dans Contes, édition intégrale, Montréal, Hurtubise HMH, 1985 [1953], p. 79-105.

Harvey, Carol J. (dir.), Paroles francophones de l'Ouest et du Nord canadiens, Winnipeg, Presses universitaires de Saint-Boniface, 2012, xv, 221 p. 
Kamba, Tchitala, L'Exilée de Makelele, Saint-Boniface, Éditions des Plaines, 2007, 64 p.

Lohka, Eileen, C'était écrit, Ottawa, L'Interligne, 2010, 104 p.

Lohka, Eileen, Miettes et morceaux, Quatre Bornes, Île Maurice, Bartholdi, 2005, 144 p.

Louder, Dean et Éric Waddell (dir.), Franco-Amérique, Sillery, Septentrion, 2008, 373 p.

Matteau, Michèle, « Où donc est la littérature? ”, Liaison, n 134, 2006-07, p. 25.

Morcos, Gamila et al, Dictionnaire des artistes et des auteurs francophones de l'Ouest canadien, Québec / Edmonton, Les Presses de l'Université Laval / La Faculté Saint-Jean, 1998, 366 p.

Moser, Marie, Counterpoint, Toronto, Irwin Publishing, 1987, 166 p.

Motut, Roger, Moissons, Edmonton, Ministère de l'Éducation de l'Alberta, 1979, 226 p.

Ouellette-Berkhout, Denise, Bonjour garde, Saint-Boniface, Éditions des Plaines, 1994, 222 p.

Ouellette-Berkhout, Denise, Collé à la peau, Saint-Boniface, Éditions des Plaines, 2012, 157 p.

Ouellette-Berkhout, Denise, Le Diamant du Jood, Saint-Boniface, Éditions des Plaines, 2007, 160 p.

Ouellette-Berkhout, Denise, Le Golé, Saint-Boniface, Éditions des Plaines, 2002, 128 p.

Ouellette-Berkhout, Denise, Quand j'aurai retrouvé mon fils, Saint-Boniface, Éditions des Plaines, 1998, 220 p.

Potvin, Claudine, Détails : contes autobiographiques, Québec, Éditions de L'instant même, 1993, 97 p.

Potvin, Claudine, Pornographies, Québec, Éditions de L'instant même, 2002, 130 p.

Primeau, Marguerite-A., Dans le muskeg, Montréal, Fides, 222 p. (réédité aux éditions des Plaines en 2005).

Primeau, Marguerite-A., Maurice Dufault, sous-directeur, Saint-Boniface, Éditions des Plaines, 1983, 200 p.

Primeau, Marguerite-A., Maurice Dufault, Vice-Principal, trad. par Maureen Ranson, Calgary, Presses Universitaires de Calgary, 2006, 222 p.

Primeau, Marguerite-A., Ol' Man, Ol' Dog et l'enfant et autres nouvelles, Saint-Boniface, Éditions du blé, 1996, 84 p. (réédité, revu et augmenté en 2004). 
Primeau, Marguerite-A., "Saint-Paul-des-Métis », dans Estelle Dansereau, Pamela V. Sing, Eileen Lohka et Paul Dubé (dir.), Alberta, village sans $\operatorname{mur}(s)$, Saint-Boniface, Presses Universitaires de Saint-Boniface, p. 7-14.

Primeau, Marguerite-A., Savage Rose, trad. par Margaret M. Wilson, Victoria, Ekstasis Editions, 1999, 146 p.

Primeau, Marguerite-A., Sauvage Sauvageon, Saint-Boniface, Éditions des Plaines, 1984, 159 p. (réédité en 2004).

Primeau, Marguerite-A., Le Totem, Saint-Boniface, Éditions des Plaines, 1988, 154 p.

Primeau, Marguerite-A., The Totem, trad. par Margaret M. Wilson, Victoria, Ekstasis Editions, 2002, 128 p.

Prud'homme, Louis-Arthur, "La littérature française au Nord-Ouest ", Mémoires de la Société Royale du Canada, Section I, Série III, vol. IX, décembre 1915, p. 247-264.

RAFA (Regroupement artistique francophone de l'Alberta), http://www. rafa-alberta.ca/, consulté le 26 février 2013.

Requier, Pierrette, Details from the Edge of the Village, Calgary, Frontenac House, 2009, 109 p.

Ricœur, Paul, Parcours de la reconnaissance : trois études, Paris, Stock, 2004, $396 \mathrm{p}$.

Saint-Pierre, Annette, «L'Ouest canadien et sa littérature ", Revue d'histoire littéraire du Québec et du Canada français, ${ }^{\circ} 12,1986$, p. 171-200.

Sing, Pamela V., «Écrire l'absence: Montréal et l'Alberta chez Marguerite-A. Primeau et Nancy Huston ", University of Toronto Quarterly, vol. 70, $\mathrm{n}^{\mathrm{0}} 3$, été 2001, p. 737-751.

Sing, Pamela V., "Stratégies de spatialisation et effets d'identification ou de distanciation dans Cantique des plaines ", dans Marta Dvorák et Jane Koustas (dir.), Vision / division, l'œuvre de Nancy Huston, Ottawa, Presses de l'Université d'Ottawa, 2004, p. 63-74.

Sing, Pamela V., "Writing the Hinterland (Back) into the Heartland: the Franco-Canadian Farouest in Two Novels by Nicolas Dickner and D.Y. Béchard ", British Journal of Canadian Studies, vol. 24, $\mathrm{n}^{\circ}$ 2, 2011, p. 221-246.

Statistique Canada, http://www12.statcan.gc.ca/census-recensement/2011/ as-sa/98-314-x/98-314-x2011003_1-fra.cfm, consulté le 26 février 2013.

Taylor, Charles, The Politics of Recognition, Princeton, Princeton UP, 1994; [en ligne] http://elplandehiram.org/documentos/JoustingNYC/ Politics_of_Recognition.pdf, consulté le 14 février 2013. 
Thériault, Joseph Yvon, Faire société. Société civile et espaces francophones, Sudbury, Prise de parole, coll. "Agora ", 2007, 384 p.

Thériault, Joseph Yvon, "À quoi sert la Franco-Amérique? ", dans Dean Louder et Éric Waddell (dir.), Franco-Amérique, Sillery, Septentrion, 2008, p. 355-365.

Toussaint, Ismène, «Littérature d'expression française dans l'Ouest canadien ", L'Encyclopédie canadienne, Historica-Dominion, 2013; [en ligne] http://www.thecanadianencyclopedia.com, consulté le 6 février 2013.

Verret-Chiasson, Jocelyne, Forêts et océans/Of Trees and Sea, Alberta, 1994, Verret-Chiasson, Jocelyne, Gens d'ici, gens d'ailleurs/People from Here and Afar, Alberta, 1996.

Verret-Chiasson, Jocelyne, J'attendrai, Saint-Boniface, Éditions des Plaines, 2003, 126 p.

Verret-Chiasson, Jocelyne, Voulez-vous danser?, Régina, Éditions de la nouvelle plume, 1996, $99 \mathrm{p}$.

Villeneuve, Gisèle, Rumeurs de la Haute Maison, Montréal, Québec/ Amérique, 1987.

Villeneuve, Gisèle, Visiting Elizabeth, Montréal, XYZ, 2004.

Wilhelm, Bernard (dir.), Sous les mâts de la prairie : anthologie littéraire fransaskoise et de l'Ouest canadien, Regina, Éditions de la Nouvelle plume, 2000, $294 \mathrm{p}$. 